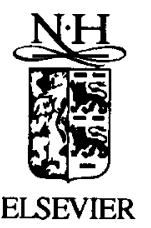

Journal of Luminescence 58 (1994) 380-387

\title{
Subwavelength spectroscopy, exciton supertips and mesoscopic light-matter interactions
}

\author{
Raoul Kopelman*, Weihong Tan, Duane Birnbaum \\ Department of Chemistry, University of Michigan, Ann Arbor, MI 48109, USA
}

\begin{abstract}
Subwavelength optical light sources and probes are leading to new devices, technologies and techniques such as near-field microscopy, spectroscopy and biochemical sensing. We have prepared nanometer light sources as small as $40 \mathrm{~nm}$ in diameter and exciton sources that are even smaller by adding various inorganic and organic crystals and molecularly doped polymers to micropipettes and nanofabricated fiber-optic tips. We demonstrate subwavelength spectroscopy with highly spatially resolved fluorescence spectral of nanocrystals (perylene) in polymeric glasses (PMMA). We also demonstrate an interfacial Kasha (external heavy-atom) effect on a perylene nanocrystal surface. We give results on near-field optics and energy transfer at interfaces of nanocrystals (anthracene or diphenyl-anthracene) and dyes (rhodamine B) or dyes embedded in Langmuir-Blodgett films. We discuss the rise of new phenomena when the light source and the sample form a combined mesoscopic system, with subsequent quantum optics effects.
\end{abstract}

\section{Introduction}

Near-field optics (NFO) has begun to gain recognition in several fields, including photofabrication, optical memories, microscopy, spectroscopy, biochemical sensing and biomedical research $[1,2]$. Near-field scanning optical microscopy (NSOM), near-field scanning optical spectroscopy (NSOS), molecular exciton microscopy (MEM) and nanofiber-optic biochemical sensors (NFOBS) are part of the newly emerging NFO arsenal $[1,2]$. Individual molecular nanocrystals and nanoglasses, DNA aggregates, quantum wells and gold colloids have been imaged optically and spectroscopically with subwavelength spatial resolution $(40-100 \mathrm{~nm})$ [1-3]. Special molecular nanostructures, such as optical nanoprobes and nano-optical biochemical

\footnotetext{
* Corresponding author.
}

sensors, are produced by near-field optical nanofabrication [4]. Not to be ignored are some underlying, poorly understood, subwavelength quantum optics (meso-optics). Where does an excitonpolariton end and a photon begin? How well can we distinguish a very long range "nonradiative" Förster energy transfer from a very short distance emission-reabsorption "radiative" energy transfer? Such questions are of much theoretical and practical interest. Coupled with the above are the microscopic phenomena of interfacial molecular (and atomic) excitation transfer and quenching. Examples are interfacial exciton transfer and Kasha (external heavy-atom) effects $[5,6]$. These have an intriguing potential for MEM and nanometerresolved spectroscopy $[2,7]$.

Conventional ("far-field") optical techniques are based on focusing elements such as a lens. This leads to a "diffraction limit" of about $\lambda / 2$ (half the 
wavelength). The realization of better resolution has led to the concept of near-field optics [1], and the realization of near-field optics is through optical nanoprobes. To form a subwavelength optical probe, light is directed to an opaque screen containing a small aperture. The radiation emanating through the aperture and into the region beyond the screen is at first highly collimated, with a dimension equal to the aperture size and independent of the wavelength of the light employed. The region of collimated light is known as the "near-field" region. The highly collimated emissive photons only occur in the near-field regime. To generate a high-resolution image, a sample has to be placed within the near-field region of the illuminated aperture. The aperture then acts as a subwavelengthsized light probe which can be used as a scanning tip to generate an image. A few techniques have been developed for nanofabricating subwavelength light and excitonic probes $[1,4,8-10]$.

In NSOM, the size of the light source determines the resolution of the image, provided that it can be scanned in the near-field region. However, the requirements of smallness and intensity are in direct conflict. Passive light sources are typically apertures letting light through, and when the size of the aperture gets to be significantly below that of a wavelength, most of the light will be diffracted or reflected back, rather than transmitted [11]. To solve this problem, an active light source, based on molecular excitons, for NSOM and MEM was proposed by Lieberman et al. [10]. Such a light source incorporates nanometer-size organic crystals or other luminescent materials at the end of a pulled micropipette with an inner diameter of a few nanometers. The basic point is to convert a passive light source into an active light and exciton source. The solution to the problem of severe light loss is to use the energy packaging capabilities of certain materials to circumvent the boundary problem of the edge of the aperture. We note that excitations can be confined to molecular and atomic dimensions under appropriate conditions [5]. Using this property of materials, one can develop a subwavelength light or exciton source by growing a suitable crystal within the subwavelength confines of a micropipette. With this approach, energy can be guided directly to the aperture at the tip instead of being allowed to propagate freely in the form of an electromagnetic wave. The excitons can be generated directly at the tip or within the bulk of the material and are allowed to diffuse to the tip via energy transfer $[5,10]$. In either case, these excitons undergo radiative decay, producing a tiny source of light at the very tip of the micropipette. The excitonic throughput is basically independent of the wavelength and is a linear function of the crosssection of the aperture [2].

Although active light sources conceptually make it possible for NSOM to achieve molecular resolution and sensitivity, it is very difficult to prepare an optical or excitonic probe of molecular size (supertip) without molecular engineering. There are a variety of methods for making supertips. In principle, the optical fiber tip can be treated chemically, so as to produce specific supertips for a variety of purposes: (1) wavelength shifters, e.g. crystallites that fluoresce to the red of the tip emission; (2) time "extenders", same as (1), utilizing prompt or delayed fluorescence or even phosphorescence; (3) highly sensitive optochemical nanosensors; (4) energy transfer supertips; (5) heavy-atom sensors. Förster energy transfer [5] or the Kasha effect (external heavy-atom effect) involves [6] interactions occurring at the interface of the supertip (its active center) and the sample (which are quantum mechanically coupled). For the highest resolution, this active center consists of a single molecule, or molecular cluster, that does the imaging. This molecule is the energy donor site for the Förster energy transfer [5], or the spin-orbit interaction site for the Kasha effect [6]. We note that a completely analogous situation is found in atomic force microscopy, where the active center is the force contact site at the tip of the supertip [2].

The aforementioned optical and excitonic probes can be used for microscopy, spectroscopy, mesoscopic light-matter interaction studies and chemical sensing. We have developed NSOM and NSOS and have prepared the smallest optical biochemical sensors $[2,3,4,7]$. NSOS can be used to obtain spectra of various nanostructures. NSOS inherits all the advantages of NSOM: its noninvasive nature, its ability to look at nonconducting and soft surfaces, and the addition of a spectral dimension, the last of which does not exist in either STM or 
AFM. The ability to obtain spectroscopic information with a nanometer-sized resolution makes NSOS very promising for the detection of fluorescent labels on biological samples and for isolating local nanometer heterogeneities in microscopic samples.

In this report, the mesoscopic light-matter interactions include energy transfer and the Kasha quenching effect. Exciton probes can be used for energy transfer studies. Excitons produced in the nanometer crystal source are the energy donor sites for a probe to sample Förster energy transfer [5], or the spin-orbit interaction sites for a probe to sample Kasha effect [6]. The Kasha effect has been observed before, only when both molecules are neighbors (or collide) inside the same phase, e.g. in liquid solution [13], because the interaction is extremely short-range, e.g. $5 \AA$ or less. We also discuss molecular light absorption in the near-field domain.

\section{Experimental}

Most of the experimental details have been published previously $[4,10,12]$. Here we briefly describe some important procedures. The first step in the probe nanofabrication process is the pulling of micropipettes and fiber-optic tips to an appropriate size and shape and the metal coating of such tips. This step is followed by crystal (or polymer) growing if active optical or excitonic probes are desired. There are two major probes used in NFO: metalcoated glass micropipettes and nanofabricated optical fiber tips $[4,8-10]$. Typical examples of them are shown in Fig. 1 as SEM micrographs.

There are two major techniques used in the preparation of active subwavelength optical and exciton probes. The first uses optical fiber probes and bonds luminescent materials covalently to the top surfaces of the probes by photo-nanofabrication, while the second uses micropipettes or nanofabricated optical fiber tips to hold crystals or doped polymers at their tips $[4,10,12]$. The nanofabricated optical fiber probes have been used successfully for the preparation of active light and exciton sources. For example, submicrometer optical fiber $\mathrm{pH}$ sensors are prepared by incorporating fluoresceinamine into an acrylamide-methylenebis (acrylamide) copolymer that is attached covalently to an activated fiber tip surface by near-field
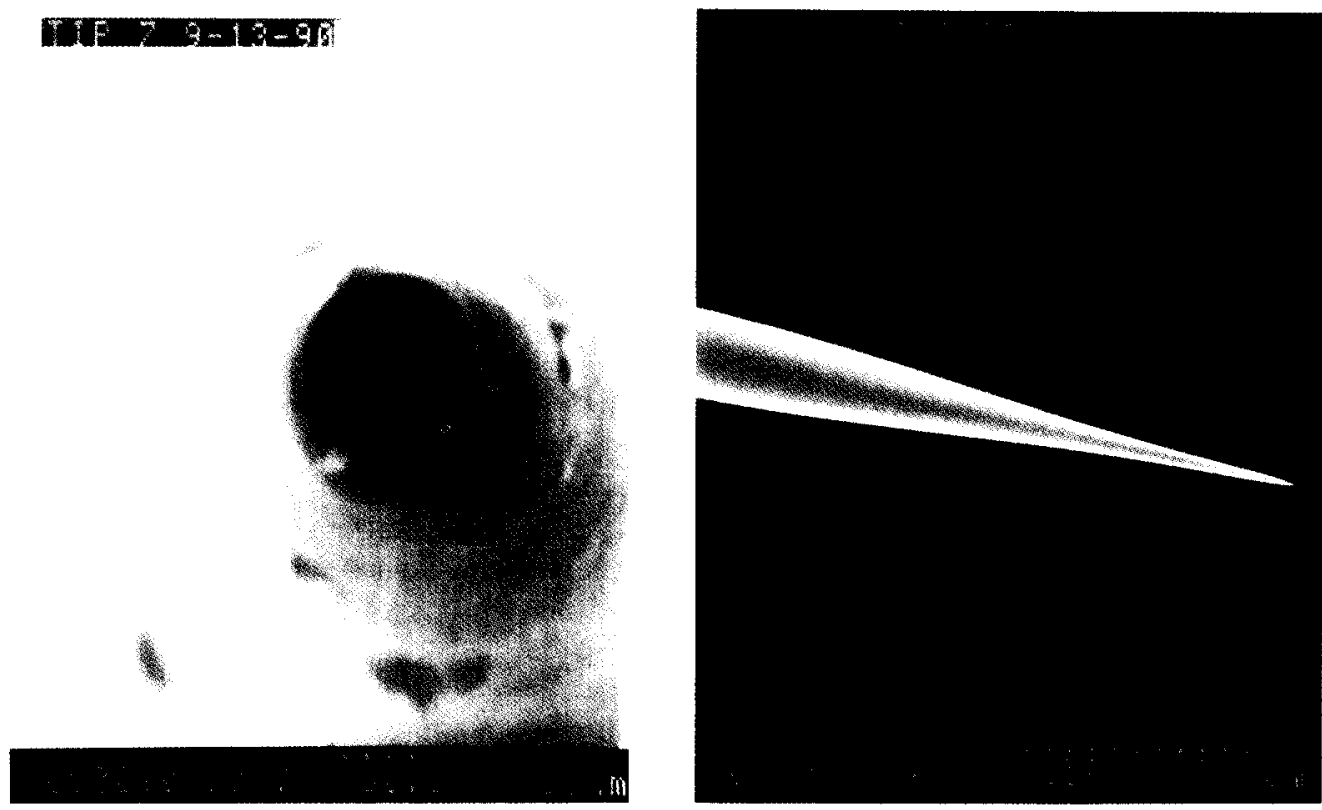

Fig. 1. SEM micrographs of nanofabricated optical fiber tip (right) and micropipette (left) used as optical nanoprobes. 
photopolymerization $[4,14]$. The same technique can be used for preparing other subwavelength light and exciton sources for NSOM, NSOS and MEM. We prepared a variety of crystals and doped polymers, such as anthracene, DPA $(9,10-$ diphenylanthracene), perylene, BASF dyes [9] and their doped PMMA or polystyrene polymers. These active probes were excited by different laser lines from UV to visible. The techniques used in the crystal growth inside micropipettes are crystal growth from solution, from melt, from vapor, from chemical reactions [12] and from other sources. Supertips are mainly prepared by dipping an active light source into another dye solution to form an active center at the tip. Optically controlled nanofabrication has also been utilized for supertip preparation. The photopolymerization is controlled by the light emanating from this near-field light source. The size of the light source and the nearfield evanescent photon profile control the size and shape of the immobilized photoactive polymer [4].

The optical and excitonic probes are mainly characterized by scanning electron microscopy (SEM) and optical measurements. Fluorescence spectra are obtained either from a $\mathbf{J}-\mathbf{Y}$ spectrometer or a microscope coupled to a spectrometer with an optical multichannel analyzer (OMA) $[12,14]$.

\section{Experimental results}

\subsection{Near-field optical nanoprobes and supertips}

Near-field optical nanoprobes can be classified into three different kinds: passive optical probes, such as coated micropipettes $[9,10]$, semi-active light sources, such as optical fiber tips [8], and active light sources, such as nanometer crystal light sources $[2,10]$. We have fabricated all three kinds of light and exciton sources. The micropipettes and optical fiber tips are easily fabricated to sizes of approximately $50 \mathrm{~nm}$, and the active light sources are even smaller. The optical fiber tips (see Fig. 1) deliver light very efficiently since most of the radiation is bound to the core until a few microns away from the tip. A randomly chosen $0.2 \mu \mathrm{m}$ optical fiber probe gives $10^{12}$ photons per second [14]. It is interesting to note that the top of a fiber tip is really very resistant to breakage. The photochemical stability for optical fiber tips is excellent. The spectral range of optical fiber tips extends from about 300 to $2000 \mathrm{~nm}$ (depending on the optical fiber quality).

Supertips have been developed with different designs: nanocrystal designer probe, polymer matrix probe and dendrimer approach [15]. In the polymer matrix approach, a polymeric matrix is attached to the optical fiber tip by spatially controlled photopolymerization [4]. This polymer supertip acts as an antenna for superimaging and supersensitivity. We have also designed a supertip made of a single symmetric macromolecule by using newly developed dendrimers $[12,15]$. Here we mainly describe the nanocrystal designer approach. This approach employs exciton conducting crystallites that absorb the light of the optic tip and convert it into excitons, which ultimately produce photons again. We have successfully grown such crystals of perylene, diphenylanthracene, etc., onto the optical tips, including fiber-optic tips and micropipettes. When the crystallite is grown at the tip of a micropipette, a fiber-optic tip is pushed deep into the micropipette, very close to the crystal tip. The crystallite acts as an antenna (compare photosynthetic antenna) that channels the excitons to an active center which acts as an exciton trap [5]. This trap collects excitation from as far as $500-1000 \AA$. The single impurity molecule (the "supertrap") creates a host "funnel" around it [2,5]. This funnel consists of host crystal molecules perturbed by the impurity ("guest") molecule. The closer the host molecule is to the trap, the lower its excitation energy. The molecules in the funnel act as exciton traps, catching the excitation from the host crystal and passing it deeper and deeper (in energy) to the deepest of them all, the supertrap.

So far the best microcrystals have been grown onto the tip of a micropipette. This tip is excited internally by a fiber-optic tip. It gives as much intensity as with luminescence, i.e. the external excitation method $[9,10]$. We prepared a DPA crystal tip, and then dipped it into different concentrations of rhodamine B solutions. The spectra of the supertip were taken with the microscope based OMA system. As shown in Fig. 2, we observed acceptable levels of luminescence intensities from a few 


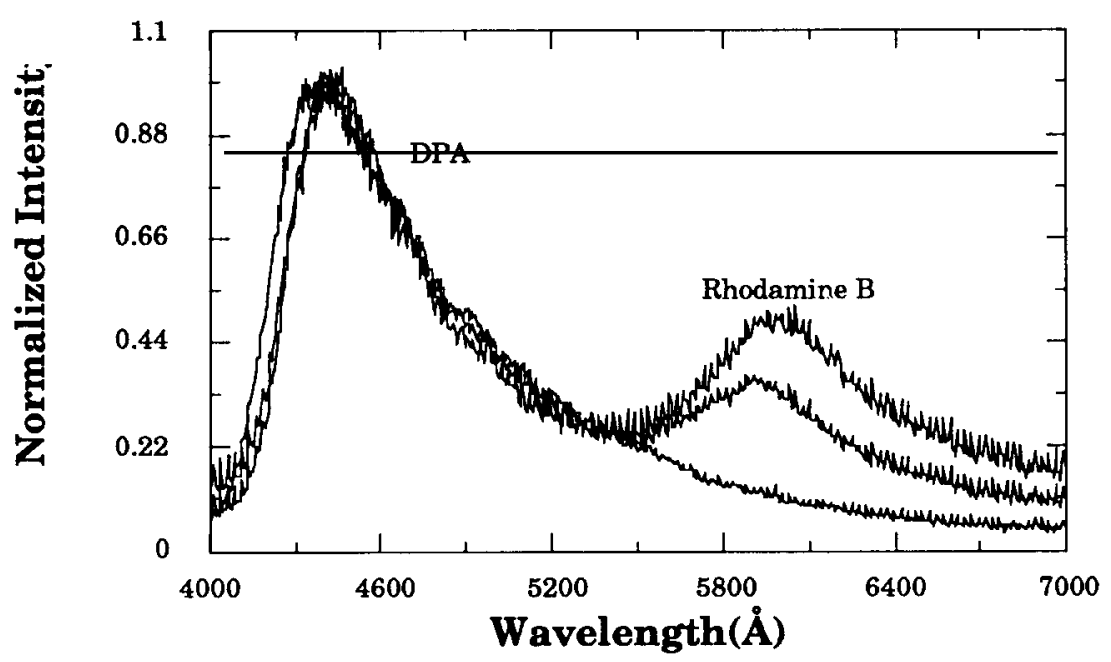

Fig. 2. Fluorescence spectrum of active centers (Rhodamine B) and supertip nanocrystal (diphenylanthracene). Top and center curves: tips dipped into rhodamine solutions of $10^{-4} \mathrm{M}$ and $10^{-5} \mathrm{M}$, respectively.

molecules of rhodamine B embedded onto the surface of a DPA crystal. From the spectra, it is clear that even when dipping into very dilute rhodamine $B$ solutions, the emission of a tiny rhodamine B active center is still detectable with our apparatus. This work clearly demonstrates that supertips could be prepared by dipping nanometer crystal tips into dye solutions. The crystal tips absorb the incident light, create excitons and emit light again (however, with their typical fluorescence spectrum) or transfer energy to the active center and emit a lower energy fluorescence. They are thus exciton supertips.

\subsection{Applications of active light sources}

The applications of active light sources are very promising. Here we demonstrate probe-to-sample energy transfer and probe-to-sample Kasha effects.

\subsubsection{Exciton tips for energy transfer}

Exciton probes have been used as the energy donor site for probe-to-sample Förster energy transfer studies. We have demonstrated the feasibility of this idea by using an anthracene or DPA crystal tip and a monolayer Langmuir-Blodgett (LB) film containing dye molecules (Di-O) [12] at low concentrations. The exciton probe is positioned on the top of the LB film and scanned in the
$Z$ direction towards the sample. From a distance of approximately $0.5 \mu \mathrm{m}$, the DPA tip is just a greenish blue light source. This emitted blue light is absorbed by the dye containing film, resulting in a yellow-orange dye fluorescence. This absorption is fairly inefficient and is barely dependent on the $Z$ distance (crystal tip to LB film), as the number of dye molecules in the beam is practically independent of $Z(Z<0.5 \mu \mathrm{m})$. However, once the DPA tip practically "touches" the LB film (coming within a Förster radius of about $5 \mathrm{~nm}$ ), the mechanism of energy (exciton) transfer dominates the LB film excitation process and much stronger yelloworange fluorescence is observed. Fluorescence intensity enhancement up to a factor of 10 has been observed when the probe physically touches the sample. A part of this enhancement could also be due to near-field light absorption (see below).

\subsubsection{External heavy-atom (Kasha) effect at inter- face}

We recall that the Kasha effect [6] is based on interatomic spin-orbit coupling, where the heavy atom has a high degree of spin-orbit coupling and the light one has very little of it. We have tested this effect by utilizing the exciton tip in probe-to-sample interfacial quenching experiments. When a solid film of perylene is in contact with a liquid solution 


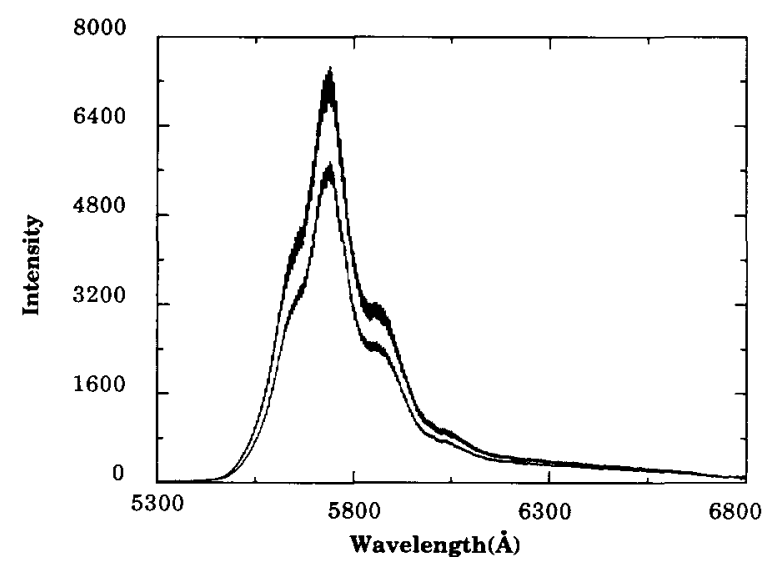

Fig. 3. Perylene tip quenching experiment. Top: perylene crystal tip immersed in $\mathrm{NaCl} /$ water; bottom: perylene crystal tip immersed in $\mathrm{Nal} /$ water.

(water) containing heavy atoms $(\mathrm{NaI})$, significant fluorescence quenching has been observed. The perylene film is at the bottom of an optical fiber tip that is scanned towards contact with the water solution (and back out again). As seen in Fig. 3, the quenching is significant. We emphasize that blank experiments, with $\mathrm{NaCl}$ water solutions, show no quenching. Furthermore, as soon as the optical fiber tip (with the perylene "supertip") is pulled out of the water solution, the fluorescence is essentially restored to its old value. This eliminates the possibility that the perylene was dissolved, or chipped off, or reacted chemically with the iodide (or formed some permanent van der Waals complex with it). To the best of our knowledge, this is the first observation of an interfacial Kasha effect. This interfacial effect occurs in the near-field zone. Note that it is owing to the presence of the quencher that the crystal surface acts as an exciton trap (but not necessarily the surface by itself).

\subsection{Near-field scanning optical spectroscopy}

With NSOS, we have studied systems of tetracene and perylene doped in PMMA. The apparatus for NSOS is similar to NSOM [1,2]. The microscopic perylene-PMMA samples were prepared by spin coating [7]. NSOS results are ob-

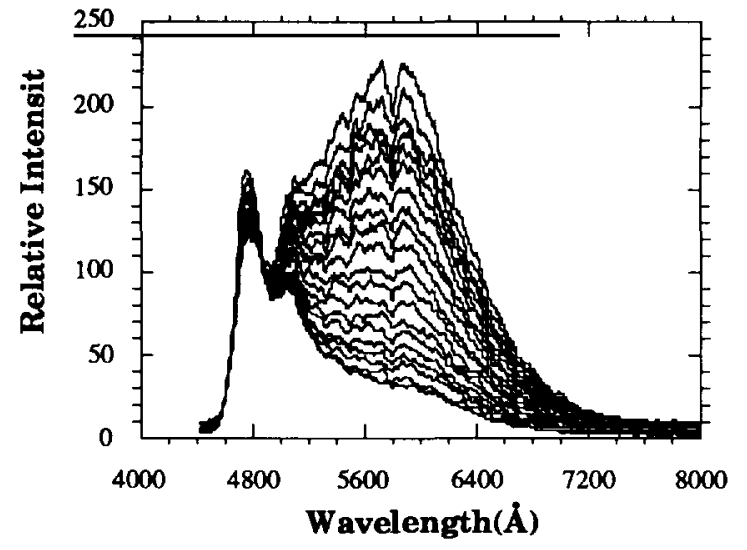

Fig. 4. Fluorescence from a blue-yellow boundary in a perylene doped PMMA spin-coated film. The peaks correspond to blue and yellow emission spectra. The size of the fiber tip was $0.5 \mu \mathrm{m}$ and the tip was scanned in increments of $400 \AA$.

tained for microcrystals of perylene which were grown on a glass slide from benzene solution.

Perylene is known to crystallize in two distinct forms [12]: the $\alpha$ form and the $\beta$ form, glowing bright green $(\beta)$ and bright yellow $(\alpha)$, respectively. To demonstrate spatial resolution by monitoring spectral features, samples of spin-coated perylene doped PMMA films were studied. After spin coating, these films were found to have regions of sharp color contrast easily observable under the microscope. That is, aggregates which fluoresce yellow are found next to blue-green fluorescent regions of the film, which are presumably due to the isolated molecule. We note that the isolated molecule fluorescence is close, although not identical, to that of the "monomeric" $\beta$ crystal $[12,13]$. Fig. 4 shows the result of bringing a fiber-optic tip within the nearfield region of the sample and monitoring the fluorescence spectrum as the tip is scanned across such an interface. It shows the results for a $0.5 \mu \mathrm{m}$ tip which was scanned across a blue-green region towards a rather large aggregate $(\sim 5 \mu \mathrm{m})$ embedded in the film. In this case, increments of only $400 \AA$ were sufficient to measure a noticeable change in the spectrum. Note that the blue-green intensity does not change as significantly since the polymeric matrix surrounds the aggregate and therefore contributes a constant background fluorescence. 


\subsection{Near-field photophysics and photochemistry}

We only summarize here some qualitative and semi-quantitative observations [12]. As stated, there is a large difference in the efficiencies of light throughput between glass micropipettes and optical fiber tips. This is in agreement with Betzig et al. [8]. In addition, several of our experiments indicate a higher per-photon molecular absorption and photopolymerization [14] in the near-field regime, compared to far-field configurations. In other words, a single molecule in the near field attenuates (e.g. absorbs) light more efficiently than in the far field, with an equal photon flux. There is, however, the question of how to define the photon flux over a region much smaller than the wavelength (see Section 4).

\section{Discussion}

We have demonstrated the ability of NSOS as a technique for obtaining high spatial resolution in a spectral dimension by studying different microscopic and nanoscopic samples. In these heterogeneous samples it is possible to isolate heterogeneities on a nanometer-size scale. Measurable changes in the fluorescence spectra are obtained for lateral increments of approximately $1 / 10$ the size of the aperture of the exciting fiber tip. This implies that it may be possible to differentiate heterogeneities at molecular sizes $(\sim 10 \AA)$, with a light source $100 \AA$ in diameter.

The problem in using micropipettes is the conflict between smallness and the light intensity. Compared to a hollow micropipette tip, a nanofabricated optical fiber tip is a "semi-active" photon tip. Generally it is orders of magnitude brighter, easily coupled to an optical source and at least as mechanically sturdy as a micropipette. At the same time, the optical properties of an active or semiactive material also lead to some advantages for optical fiber tips. Using a classical optics description, we note that the higher refractive index $(n)$ of the fiber material reduces the photon wavelength inside it to $\lambda=\lambda_{0} / n$, compared to the wavelength in vacuum or air $\left(\lambda_{0}\right)$. This reduces significantly the diffraction of the light at the orifice. In principle, as $i$ approaches the optical absorption of the dielectric, $n$ increases, and eventually becomes a complex quantity [2]. We also note that the Einstein ab. sorption coefficient [16] is proportional to $(v / c)^{3}$, i.e. to $\lambda^{3}$. The reduction of $\lambda$ in a high refractive index material should thus not only reduce the back-scattering, mentioned above, but also increase the optical absorption cross-section (as well as other optical cross-sections). This may be due to an increase of higher-order terms, such as quadrupole transition moments [16]. Alternatively, one can use a quantum approach and consider the excitonpolariton resonance or quasi-resonance $[17,18]$. Thus, the optical fiber tip exhibits a crossover with wavelength from a passive to an active class of photon tip. This may be one of the major reasons for the optical fiber tip's high light throughput.

The concept of active light source enables a totally new mode of NSOM, based not on the blocking or absorption of photons but rather on direct quenching of the energy quanta that otherwise would have produced photons. For instance, a thin, localized gold film (or cluster) can quench an excitation (or exciton) that would have been the precursor of photons. Furthermore, a single atom or molecule on the sample could quench (i.e. by energy transfer) the excitations located at the tip of the light source. For simplicity, we assume that the active part of the light source is a single atom, molecule or crystalline site, serving as the "tip of the tip". This quenching energy transfer from the excitation source's active part (donor) to the sample's active part (acceptor) may or may not qualify technically as an NSOM technique. However, it is the best hope, currently, for single atom or molecule resolution and sensitivity, as explained below. Semantically, we could call this technique quantum optics microscopy. Alternatively, it has been called molecular exciton microscopy (MEM) [2]. MEM is conceptually quite similar to STM. The excitons "tunnel" from the tip to the sample. However, there is no driving voltage or field. Rather, it is the energy transfer matrix element which controls the transfer efficiency. Its unusual matrix elements allow for the highest sensitivity to distance, higher than that of STM and comparable to that of AFM [2]. In addition, the most striking result of this direct energy transfer is its ultrahigh sensitivity to isolated 
or single molecular chromophores. The quantum optics energy transfer is highly efficient within the range of the "Förster radius". Thus, a single excitation could be "absorbed" by the sample acceptor. In contrast, based on the Beer-Lambert law, about a billion photons are needed [2] to excite a single acceptor in the absence of other acceptors. Furthermore, as the distance range is limited to about $8 \mathrm{~nm}$ for the direct energy transfer, molecular exciton microscopy is as much a near-field technique as STM or AFM, i.e. it is very sensitive in the single digit $\mathrm{nm}$ range and much less sensitive beyond $10 \mathrm{~nm}$. However, in combination with conventional NSOM, the range can be extended to about $100-200 \mathrm{~nm}$ (and, adding far-field optical microscopy, to microns and beyond). It also allows for a much more universal range of samples, from metal spheres and clusters to soft, in vivo biological units.

\section{Summary}

Different subwavelength light and exciton probes have been prepared by micropipettes and nanofabricated optical fiber tips. A battery of nanotechniques have been developed to design and fabricate these nano-optic and excitonic probes. Using these subwavelength probes, we studied both probe-to-sample energy transfer and probe-tosample interfacial Kasha quenching effects. Also, we have demonstrated the ability of NSOS as a technique for obtaining nanometer-size spatial resolution by studying different microscopic and nanoscopic samples. Furthermore, supertips have been prepared, and their development and optical properties have been discussed. The large variety of nanoprobes promises many potential applications for near-field optics.

\section{Acknowledgements}

We thank our colleagues, Dr. Zhong-You Shi, Mr. Steve Smith and Dr. Renato Zenobi for helpful discussions. This work is supported by NSF grant DMR-9111622.

\section{References}

[1] D.W. Pohl, in: Advances in Optical and Electron Microscopy, eds. C.J.R. Sheppard and T. Mulvey (Academic Press, London, 1990) pp. 243-312.

[2] R. Kopelman and W. Tan, in: Spectroscopic and Microscopic Imaging of the Chemical State, ed. M.D. Morris (Marcel Dekker, New York, 1993) pp. 227-254.

[3] S. Smith, E. Monson, G. Merrit, W. Tan, D. Birnbaum, Z. Shi, B. Thorsrud, C. Harris, H.T. Grahn, K. Ploog, R. Merlin, B. Orr, J. Langmore and R. Kopelman, SPIE 1858 (1993) 13.

[4] W. Tan, Z.-Y. Shi, S. Smith, D. Birnbaum and R. Kopelman, Science 258 (1992) 778.

[5] A.H. Francis and R. Kopelman, Topics in Applied Physics, Vol. 49, Laser spectroscopy of solids, eds. W.M. Yen and P.M. Selzer (Springer, Berlin, 1986; 2nd ed.) p. 241.

[6] M. Kasha, J. Chem. Phys. 20 (1952) 71.

[7] D. Birnbaum, S. Kook and R. Kopelman, J. Phys. Chem. (1993).

[8] C. Betzig, J.K. Troutman, T.D. Harris, J.S. Weiner and R.L. Kostelak, Science 251 (1991) 1468.

[9] A. Lewis and K. Lieberman, Anal. Chem. 63 (1991) $625 \mathrm{~A}$.

[10] K. Lieberman, S. Harush, A. Lewis and R. Kopelman, Science 24 (1990) 59.

[11] A. McDonald, IEEE Trans. Microwave Theory Tech. MTT-20 (1972) 698

[12] W. Tan, PhD Thesis, University of Michigan, Ann Arbor, MI (1993).

[13] M. Pope and E. Swenberg, Electronic Processes in Organic Crystals (Oxford University Press, New York, 1982).

[14] W. Tan, Z.-Y. Shi and R. Kopelman, Anal. Chem. 64 (1992) 2985.

[15] Z. Xu, Z.-Y. Shi, W. Tan, R. Kopelman and J.S. Moore, Polymer Preprints 33 (1993) 130-131.

[16] D. Bohm, Quantum Theory (Prentice-Hall, New York, 1951).

[17] V.M. Agranovich and M.D. Galanin, Electronic Excitation Energy Transfer on Condensed Matter (NorthHolland, Amsterdam, 1982).

[18] J. Aavikso, A. Freiberg, J. Lipmaa and T. Reinot, J. Lumin. 37 (1987) 313. 